\title{
Critical Neuronal Models with Relaxed Timescale Separation
}

\author{
Anirban Das ${ }^{1, *}$ and Anna Levina ${ }^{2,3,4, \dagger}$ \\ ${ }^{1}$ Department of Mathematics, Pennsylvania State University, McAllister Building, State College, \\ Pennsylvania 16802, USA \\ ${ }^{2}$ University of Tübingen, Max-Planck-Ring 8, 72076 Tübingen, Germany \\ ${ }^{3}$ Max Planck Institute for Biological Cybernetics, Max-Planck-Ring 8, 72076 Tübingen, Germany \\ ${ }^{4}$ Bernstein Center Tübingen, Max-Planck-Ring 8, 72076 Tübingen, Germany
}

(Received 11 June 2018; revised manuscript received 12 February 2019; published 28 June 2019)

Power laws in nature are considered to be signatures of complexity. The theory of self-organized criticality (SOC) was proposed to explain their origins. A long-standing principle of SOC is the separation of timescales axiom. It dictates that external input is delivered to the system at a much slower rate compared to the timescale of internal dynamics. The statistics of neural avalanches in the brain was demonstrated to follow a power law, indicating closeness to a critical state. Moreover, criticality was shown to be a beneficial state for various computations leading to the hypothesis that the brain is a SOC system. However, for neuronal systems that are constantly bombarded by incoming signals, the separation of timescales assumption is unnatural. Recently, it was experimentally demonstrated that a proper correction of the avalanche detection algorithm to account for the increased drive during task performance leads to a change of the power-law exponent from 1.5 to approximately 1.3, but there is so far no theoretical explanation for this change. Here, we investigate the importance of timescale separation, by partly abandoning it in various models. We achieve it by allowing for an external input during the avalanche, without compromising the separation of avalanches. We develop an analytic treatment and provide numerical simulations of a simple neuronal model. If the input strength scales as one over the network size, we call it a moderate input regime. In this regime, a scale-free behavior is observed; i.e., the avalanche size follows a 1.25 power law, independent of the exact size of the input. For a perfectly timescale separated system, an exponent of 1.5 is observed. Thus, the universality class of the system is changed by the external input, and the change of the exponent is in good agreement with experimental observations from nonhuman primates. We confirm our analytical findings by simulations of the more realistic branching network model.

DOI: 10.1103/PhysRevX.9.021062

Subject Areas: Biological Physics, Complex Systems, Statistical Physics

\section{INTRODUCTION}

A variety of natural systems provide observations that follow power-law statistics, possibly with an exponential cutoff [1-3]. For example, a power-law distribution for activity propagation cascades (so-called neuronal avalanches) is reported in numerous neuronal systems in vitro and in vivo and at various spatial and temporal scales [3-8]. In many cases, the appearance of power-law statistics is connected with closeness to the critical point of a secondorder (continuous) phase transition. For the brain, the claim that a power-law observation points to a closeness to critical

\footnotetext{
*anirban.das.psu.math@gmail.com

†anna.levina@uni-tuebingen.de
}

Published by the American Physical Society under the terms of the Creative Commons Attribution 4.0 International license. Further distribution of this work must maintain attribution to the author(s) and the published article's title, journal citation, and DOI. states is additionally supported by the observation of stable exponent relations [9], finite-size scaling [10], and shape collapse [9]. Models of criticality therefore began being used for studying the brain. Additional reasoning for this connection comes from observations that criticality brings about optimal computational capabilities [11,12], optimal transmission and storage of information [13], and sensitivity to sensory stimuli $[14,15]$. In spite of these reasons for real systems to be close to criticality, there are many ongoing debates about models and data-analysis techniques [16] used to demonstrate this fact $[17,18]$.

The concept of self-organized criticality (SOC) was proposed [19] as a unified mechanism for positioning and keeping systems close to criticality. SOC models have emerged as the flagship vehicle for modeling criticality as an operational state of the brain network, because they eliminate the necessity to endogenously tune parameters. For a system consisting of many interacting nonlinear units, the general theory prescribes conditions necessary for exhibiting self-organized criticality. First, it should obey 
local energy-conservation rules [20], and, second, the timescale of the external drive should be separated from the timescale of interactions. It implies that no external input is delivered to the system before it reaches a stable configuration. The intuition behind the timescale separation condition can be summarized as follows: Consider that there is a macroscopic scale at which the external energy is applied and a microscopic scale for activity propagation through the interacting units. When the two scales are comparable, the frequency of the drive becomes a factor that can be tuned by some moderating party. In the limit, as the frequency of macroscopic events implodes to zero, global supervision ceases, and a self-organized system emerges [21-24].

Theoretical studies of avalanchelike propagation in networks were initiated before the first experimental results. The initial models were simple [25-29]; later, following experimental findings, more biologically realistic models were developed [30-34]. A detailed review of studies of criticality in biological systems through experiments and models may be found in Ref. [35].

Most current models and evaluation techniques of experimental results rely on the binning procedure for the definition of avalanches. Namely, the total time of a real or digital experiment is split into short intervals (bins). Empty bins are considered to be pauses between avalanches, which span bins containing at least one active unit. A rare exception is the leaky integrate-and-fire neuronal model by Millman et al. [36]. There, the avalanche is defined by following the time course of activity propagation on the known network. However, recently it was demonstrated [37] that the classical procedure of binning will not reveal any critical statistics for this model, and a neutral theory could explain the observed power laws without necessitating an SOC model. The usage of binning for data analysis from neuronal recordings $[3,38]$ implicitly relies on the assumption of timescale separation. Otherwise, the bin size would be a stiff [39] control parameter for a data analysis that can change the outcome of the evaluation. However, in neuronal systems, inputs are constantly present, and there is no chance for a strict separation of external input from the internal dynamics.

We investigate here the effects of relaxing the timescale separation condition by implementation of an input process during avalanches and observing the consequent avalanchesize distribution. Our emphasis here is on models that, on the one hand, present signatures of criticality even when the drive is absent and, on the other hand, try to mechanistically explain the power-law generation in activity propagation. Without this restriction, a power-law statistics is observed in driven stochastic processes [40-42]. In avalanching models, additional input to the system generally has two effects: First, the avalanches increase due to the input and follow-up firing; second, the avalanches are "glued together"; namely, the input connects avalanches that would have otherwise occurred separately. For the systems that are driven by a constant input, the definition of criticality is possible by the estimation of the branching ratio [43]. However, in this case, a typical binning-based avalanche analysis might not reveal a critical state, because both aforementioned effects are present simultaneously and separating the avalanches becomes impossible. A recent study [44] numerically demonstrated that changing the binning according to the firing rate reveals critical dynamics during task performance, when additional input on top of ongoing activity is expected. Here, we investigate analytically how criticality can be preserved even if an external drive is added to the system.

As a first step towards the understanding of timescale separation, we allow for external input during avalanches without compromising their separation. We develop an analytic treatment and provide numerical simulations for a simple neuronal model. We show that the power-law scaling feature is preserved; however, even moderate external input leads to a change in the slope of the avalanche-size distribution. The same critical exponents are persistent throughout a range of values of the input. Therefore, we prove that the rate of input is not taking the role of a tuning parameter. The analytic results are reproduced when simulating more realistic branching models, where input is added at a constant rate.

\section{MODELS}

We use the following two models. The branching model (BM) $[15,45]$ is a standard model to study an abstract signal propagation that serves as a simplified model for neuronal avalanches. For our studies, we equip the standard BM with an input process. Unfortunately, the BM does not allow for a complete analytic description. To overcome this difficulty, we first introduce a simpler levels model (LM). We carry out a rigorous mathematical study of the LM and then verify through simulations that similar results also hold for the BM. In the limit, as the system size grows to infinity, both the $\mathrm{LM}$ and the $\mathrm{BM}$ are well approximated by branching processes $[46,47]$.

\section{A. The levels model}

The LM without input is inspired by the simple network model of perfect integrators [29]. The neuronal avalanches produced by the model are shown to exhibit critical, subcritical, and supracritical behavior depending on the control parameter, similar to experimental observations in cortical slices and cultures [3]. The different modifications of the LM were extensively studied mathematically [47-50]. The version used here was introduced in the context of dynamical systems to prove ergodicity of avalanche transformations [50] (see the Appendix A). The main difference between the original biophysical model [29] and the LM is that the latter does not allow self-connections. However, when parameters are rescaled 


\begin{tabular}{|c|c|c|c|c|}
\hline \multicolumn{5}{|c|}{ Energy levels: } \\
\hline 7 & घロேमロ & 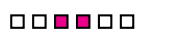 & वேमロप् & मேमロ \\
\hline 6 & ロロロロロロ & ロロロロロ & ロロロロロロ & ロロロロロ \\
\hline 5 & ロロロロロ & ロロロロロロ & ロロロロロロ & ロோロ・ \\
\hline 4 & ロ・ロロロロ - & 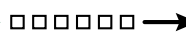 & ロロロロロロ - & प्वप्व \\
\hline 3 & ロロロロロロ & ロロロロロロ & ロロロロロロ & ロோロロ \\
\hline 2 & मロप्र & 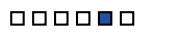 & मロप्र & 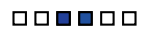 \\
\hline$M=1$ & 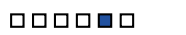 & 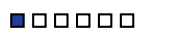 & मロघमロ & 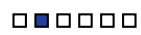 \\
\hline Neurons: & 123456 & 123456 & 123456 & 123456 \\
\hline
\end{tabular}

FIG. 1. Schematic representation of the levels model without external input, for $N=6$ neurons with $M=7$ energy levels. The avalanche size is 4 , and the avalanche duration is 3 .

to accommodate for changed connectivity, distributions of avalanche sizes and durations are the same in both models.

The LM consists of a fully connected network of $N$ units, where each unit $j$ is described by its energy level $E_{j} \in\{1, \ldots, M\}$. Connections are defined such that receiving one input changes the energy level by 1 . In the language of neuronal modeling, $E_{j}$ is the membrane potential and the connection strength is set to 1 . If neuron $j$ reaches threshold level $M$, it fires a spike, and then we reset it: $E_{j} \mapsto 1$. All neurons $k$ that are connected to $j$ such that $E_{k}<M$ are updated: $E_{k} \mapsto E_{k}+1$. After firing the spike, a neuron is set to be refractory until activity propagation is over. We initialize the model by randomly choosing energy levels of all neurons from independent copies of a uniform distribution on $[1, M]$.

After initialization, all neurons in the energy level $M$ spike, which increases the energy levels of connected neurons. If, as a result, more neurons reach the level $M$, then they are in turn discharged and so on, until the activity stops. This propagation of activity we call an avalanche, and the number of neurons fired is its size. The progression of the avalanche in a system with $N=6$ and $M=7$ is demonstrated in Fig. 1.

We introduce the external input to be proportional to the size of the activity propagation that would take place in the timescale-separated regime, i.e., without input. As before, each neuron is initialized at a random energy level. Then activity propagates deterministically until none of the neurons can fire, which concludes the initial stage of the avalanche. Afterwards, we determine the number of additional inputs to be added to the system. This number is chosen at random, depending on the size of the avalanche in the initial stage. The additional inputs allow the avalanche to continue further. If $o$ is the number of neurons fired in an avalanche, we additionally activate $r$ among the remaining $N-o$ neurons. Here, $r$ is a random number drawn from a binomial distribution $B(o, \phi)$. The parameter $\phi \in[0,1)$ represents the rate of the external input; i.e., $\phi$ is the average number of inputs delivered during an avalanche of size 1. After these $r$ additional firings, more neurons may reach the energy level $M$, resulting in a second cascade of firings. We constrain the neurons to fire only once and then go into the refractory period until the next avalanche. Thus, the process stops after a maximum of $N$ discharges. We study the dependence of the avalanche-size distribution on the strength of the input. We use $A_{N, \phi}$ to denote the random variable that counts the avalanche size. When $\phi=0$, we have the no-external-input regime. Our model without input possesses an Abelian property [51]; namely, the size of avalanches does not depend on the order in which neurons are discharged. After the delivery of the input, the resting dynamics is also Abelian. This version of the LM also proves to be mathematically tractable (see Appendix B).

\section{B. The branching model}

The BM consists of $N$ neurons connected as an ErdósRényi random graph with probability of connection $p^{\text {conn }}$. This model is used to understand criticality [45] and its benefits [15] in neural systems and is also employed in many modeling investigations of neuronal avalanches $[4,14,52]$. Every edge in the network is assigned a weight $p_{i j}=\sigma /\left(p^{\text {conn } N}\right)$. As a result, the average sum of all outgoing weights equals $\sigma$. Each node denotes a neuron that can be in one of $n$ states, and $c_{i}(t)$ denotes the state of the $i$ th node at time $t: c_{i}=0$ indicates a resting state, $c_{i}=1$ is the active state, and $c_{i}=2, \ldots, n-1$ are the refractory states. All states except for the active state are attained by the deterministic dynamics: If $0<c_{i}(t)<n-1$, then $c_{i}(t+1)=c_{i}(t)+1$, and if $c_{i}(t)=n-1$, then $c_{i}(t+1)=0$.

For every node $i$, the excited state $c_{i}(t)=1$ can be reached only from the resting state [i.e., $c_{i}(t-1)=0$ ] in one of the following circumstances: (i) If a neighbor $j$ is active at time $t-1$, then, with probability $p_{j i}, i$ will get activated at time $t$; (ii) if $i$ receives an external stimulus, the probability of this is $\phi / N$. We initiate the network with all nodes in a silent state, and then an external stimulus can trigger the activity propagation (avalanche). If at a particular time step no units are in the active state, the avalanche is considered to have terminated. We record the distribution of avalanche sizes (measured in the number of activations during one avalanche) and durations (measured as the time steps taken until activity dies out).

It was shown $[15,45]$ that, in the model without input, a network can exhibit different dynamical regimes depending on the value of the parameter $\sigma$ (called the branching parameter): When $\sigma<1$, the activity dies out exponentially fast; for $\sigma>1$, there is a possibility for indefinite activity propagation. In the critical regime, obtained for $\sigma=1$, activity propagation size $s$ is distributed as a power law with an exponent of 1.5. An external input applied to a sparsely connected branching network with Boltzmann distribution of synaptic strength is demonstrated to exhibit quasicriticality [53]. Here, we consider a uniformly connected random network and demonstrate how additional input changes scaling in the avalanche-size distribution.

\section{RESULTS AND INTERPRETATION}

\section{A. Input impact in LM}

In the "no-external-input regime," critical behavior is observed when $M=N$. In this case, the avalanche-size 
probability scales as a power law, i.e., $P\left(A_{N, 0}=k\right) \sim C_{1} k^{-1.5}$ [50]. For the rest of the article, we consider $M=N$, which still serves as the critical value of the parameter in the "driven" case, with $\phi>0$.

We let $o$ denote the size of the avalanche that would have been observed without an external drive, and then there will be on average $o \times \phi$ inputs. When $\phi=o(1 / N)$, we can show analytically that $P\left(A_{N, \phi}=k\right) \sim C_{2} k^{-1.5}$. This is the small input regime; the perturbation of the system is not strong enough to induce significant changes in the dynamics. This result demonstrates the stability of the classical models. At the other end of the spectrum, we could force a fraction of the neurons to fire as a result of the external input. Thus, $\phi=\Theta(N)$, where $\Theta$ is taken as in the Bachmann-Landau notation [54]. In such a case, we can show that $A_{N, \phi}$ converges in distribution to a normal variable, as $N \rightarrow \infty$ (see Appendix C). Essentially, the immense external input in this regime (named the large input regime) reduces the neuronal activity to "noise."

The most interesting case is the moderate input regime, where $\phi=\Theta(1)$. In this case, we can mathematically derive (see Theorem B2) the following result as $k$ grows to infinity:

$$
P\left(A_{N, \phi}=k\right) \sim C_{3} k^{-1.25} .
$$

We verified Eq. (1) by simulating a finite LM with $N=10^{5}$ neurons and inputs of varying strength. As expected, the approximate 1.5 power law (here, it is 1.45 , and it approaches 1.5 in the limit of large systems) is transformed by the input into the 1.25 power law (see Fig. 2). Also, we numerically test the avalanche duration distribution, i.e., the number of time steps during an

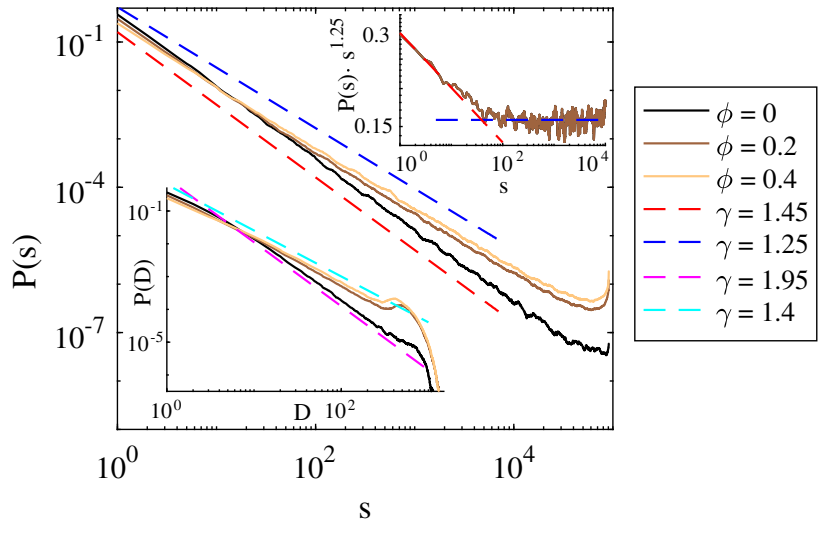

FIG. 2. Avalanche-size $(s)$ distributions in the LM with various input strengths. The lower inset shows the corresponding duration $(d)$ distributions which also change their exponent. The upper inset shows an enlargement of the distribution for $\phi=0.2$ multiplied with $s^{1.25}$ to flatten the distribution and both powerlaw fits. Input strength $\phi$ and the power-law exponents $(\gamma)$ of the lines are indicated in the legend. $N=M=10^{5}$. avalanche. Both observables deviate from the power law in the very tail because of the finite system size and the restriction on double activation. Except for these deviations, the numerical simulations support analytic results.

In the moderate input regime, in spite of the input during avalanches, power-law scaling is preserved for both avalanche size and duration distributions. However, the power-law exponent is changed. Surprisingly, as long as $\phi=\Theta(1)$, the power-law scaling is preserved, and the limiting exponent remains equal to 1.25 . This result means $\phi$ does not need to be externally tuned to achieve criticality.

\section{B. Finite-size effects and numerical results for $\mathbf{L M}$}

A scaling relationship given by Eq. (1) is valid for any given $\phi$ if $N$ and $k$ are both large enough and $k / N$ is small enough. To define a more precise parameter relationship that allows us to test results in simulations, we devise a sufficient but not necessary condition for Eq. (1) to hold. We require $k$ to satisfy

$$
N \geq k^{2} .
$$

And we require $\phi$ to satisfy, for some positive $\delta$,

$$
e^{-[\phi \log (N)]^{2}} \leq N^{-0.5-\delta} .
$$

For any $N$ and $\phi$ satisfying Eq. (3) if $k$ is small, we get $P\left(A_{N, p}=k\right) \sim C_{4} k^{-1.5}$ (the same as for no-input systems), and as $k$ grows larger we get $P\left(A_{N, p}=k\right) \sim C_{3} k^{-1.25}$, indicating multifractal behavior [55]; see Fig. 2, inset. Approximating the stochastic input by its average, we show that as long as $k \leq \phi^{-2}$ we have $P\left(A_{N, p}=k\right) \sim C_{4} k^{-1.5}$. The simple intuition behind the multifractal behavior is that, for very small avalanches, there is a substantial probability not to receive any external inputs. Thus, the 1.5 power law characteristic of traditional models with a separation of timescales is still visible.

We simulate the LM for different input strengths and observe a good agreement with our analytic results (Fig. 5, solid lines). Aberrant behavior for large avalanche sizes is due to the finite size of the system and the imposed condition that no avalanche can be larger than the system size. The theoretical prediction for the onset of the 1.25 power-law scaling is indicated by the magenta line, which too is in good agreement with numerical observations.

\section{Branching model with input}

A BM without external input corresponds to the situation where $\phi=0$; in such a scenario, the probability distribution for avalanches follows a 1.5 power law [15]. Here, we discuss the changes in the avalanche-size distribution upon adding a moderate input. A useful characteristic of the LM is that the avalanche can be separated into two stages: an original avalanche (preavalanche) and the aftershock 

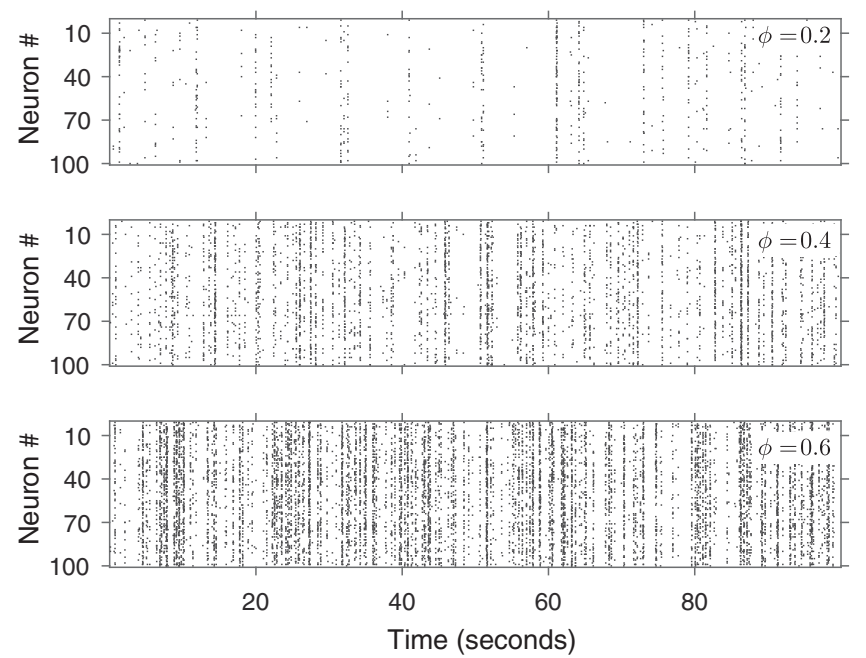

FIG. 3. Raster plot of the activity for different input strengths (BM). We randomly selected 100 out of $N=10^{5}$ neurons.

avalanche that is triggered by external inputs. Although this feature makes the LM analytically tractable, it also makes its construction seem contrived. In contrast, in the BM, external input is added at a fixed rate throughout the entire simulation time (see Fig. 3).

In the moderate input regime of the BM, for any suitable strength of the external signal, the exponent changes from 1.5 to 1.25 (Fig. 4). For large avalanches, finite-size effects observed previously in the LM are enhanced by the possibility for the system to get additional external input during the aftershock.

For the BM, the input is delivered at a constant rate, and, thus, the number of external inputs during an avalanche is proportional to the duration of the avalanche, while in the LM the input is proportional to the size of the preavalanche. However, both systems show very similar avalanche-size distributions for various input intensities (Fig. 5). Let $t_{r}$

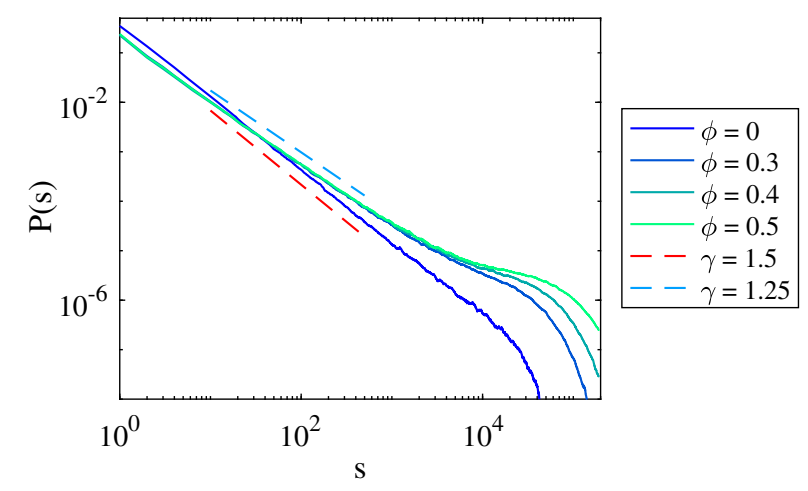

FIG. 4. Avalanche-size distributions in the branching model with various input strengths. Input strength $\phi$ is indicated in the legend. $N=10^{5}, n=10$, and $\sigma=1$. Distributions for $\phi>0$ are shifted such that they all coincide for $s=100$.

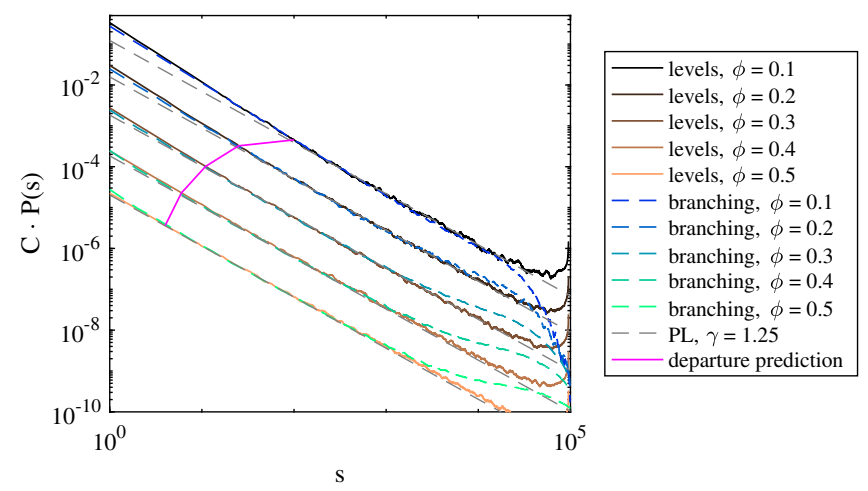

FIG. 5. Avalanche-size distributions in the levels model and branching model with various input strengths. Input strength $\phi$ and the model type are indicated in the legend. The magenta line indicates the analytic prediction for the onset of the power law with exponent -1.25 . For both models, we take $N=10^{5}$. To improve visibility, the distributions are shifted by multiplication with $c_{\phi}=10^{-10 \phi+1}$. Separate figures for the BM and the LM can be found in Appendix D.

denote the transition time between the power law with exponent 1.5 and the power law with exponent 1.25 . We observe that $t_{r}$ for the BM is roughly the same as for the LM, where we have analytic arguments showing $t_{r} \approx \phi^{-2}$ (Fig. 5). As our results for the BM rely on a statistical evaluation of the closeness to the 1.25 power law, we check whether the alternative models, such as the truncated lognormal [56], exponential [57], log-polynomial [58,59], or thresholded Ornstein-Uhlenbeck process [60], better describe the data. To this end, we perform a maximumlikelihood optimization for parameters of both truncated power-law and log-normal fit to the same interval of the avalanche-size distribution. In all cases, the power-law model has a larger likelihood, and in most cases we cannot reject a power-law hypothesis $[61,62]$. For a more detailed discussion of the statistical method, see Appendix E.

\section{CONCLUSION}

Models exhibiting criticality are classified into universality classes based on power-law exponents. Quantitative characteristics of various emergent properties in critical systems belonging to the same universality class are found to be similar (see Ref. [63]). By introducing external input to models from the 1.5 exponent universality class, we have changed them to models with characteristic power-law exponent 1.25. Although a 1.25 exponent is more seldom than the ubiquitous 1.5 exponent, the former has been observed in several models, for example, in models for slow crack growth in heterogeneous materials [64], driven elastic manifolds in disordered media [65], fracturing processes under annealed disorder [66], mesomodels of amorphous plasticity [67], and randomly growing networks [68]. 
A recent experimental study [44] has shown that taskrelated cortical activity is comprised of neuronal avalanches with an exponent very close to our prediction. By carefully accounting for the increase in the firing rate during task performance, the power law was observed to change from 1.5 to 1.3 . The observed increase in the firing rate and nLFP rate in the premotor cortex during the task performance can be attributed to the increased input from the sensory and higher areas needed for motor planning. There is a significant difference between models we consider and data analysis from the recordings. Whereas in our case the ground truth about splitting the activity into avalanches is known, for the recorded data the binning procedure influences the split significantly. However, the closeness of exponents obtained from the interpretation of experimental recordings to our analytic results suggests that the adaptive binning procedure is the right choice to capture underlying dynamics.

We demonstrated that for an input-driven system a multifractal characteristics of the avalanche-size distribution can be expected. Indeed, analytic approximations show that the 1.5 power-law exponent known from the analysis of spontaneous activity persists when $k \leq \phi^{-2}$. This persistence was not seen in Ref. [44], where the data collapsed to a single power law. On one hand, this discrepancy could come from the difference in avalanche detection mechanisms. On the other hand, it is possible that a large input during the task shifts the transition point between different scalings towards small avalanche sizes. Thus, an onset of the 1.25 power-law scaling is very close to 1 , making it undetectable in the data. This hypothesis can be tested in experiments on stimulated cortical slices [14] by varying the stimulation strength and detection algorithm. The fact that the same critical exponents persist throughout the moderate input regime suggests that the values of the critical exponents are not determined by the nature of the task. We predict the same exponent independently on the involvement of the animal and difficulty of the task.

There are many open questions related to the present investigation. The most important one is: How does the full elimination of timescale separation change the avalanche statistics? In the present contribution, we did not allow for avalanches to be mixed and run parallel to each other. With simultaneous avalanches, there is no clear understanding of how one should attribute each event to any particular avalanche. Information-theoretical measures were proposed to distinguish spikes from different avalanches [69]. So far, the most established way to study a possible "melange of avalanches" $[38,43]$ is to use binning and identify empty bins to determine pauses between the avalanches. This procedure results in different power-law exponents for different bin sizes [3], unless the system exhibits a true timescale separation [4].
Abandoning timescale separation introduces the dependence of avalanche distribution on a binning procedure. The logical hypothesis is that input during the avalanches should result in smaller power-law exponents, as larger events now become more probable. Although the direction of the exponent change is easily predictable, the fact that input preserves the power-law scaling is still surprising. Here, we demonstrated this effect analytically for the levels model and numerically for a branching network, but the general direction of change will remain the same for models from other universality classes. If we additionally allow for gluing of avalanches together, it might lead to selecting a smaller bin size than is suggested by the activity propagation timescale. This selection, in turn, will result in the cutting of avalanches into smaller pieces and increasing the power-law exponent, which might be a reason behind the observation of exponents above 1.5 and even around 2 for neuronal spiking data [9] and LFP in ex vivo turtle recordings [70]. Here, we consider a fully connected or sparse randomly connected networks. However, it has been shown [71] that network topology has an effect on power laws. Thus, driven models with specifically structured connections can produce distributions with exponents significantly larger than 1.25 . Our result is a first step towards understanding the diversity of power-law exponents reported in the neuronal data.

\section{ACKNOWLEDGMENTS}

A. L. received funding from the People Program (Marie Curie Actions) of the European Union's Seventh Framework Program (FP7/2007-2013) under REA Grant Agreement No. [291734]. A. L. received funding from a Sofja Kovalevskaja Award from the Alexander von Humboldt Foundation, endowed by the Federal Ministry of Education and Research. We also thank Professor Manfred Denker for his advice and insight.

\section{APPENDIX A: MATHEMATICAL SETUP}

In this section, we build a mathematical formalization for the LM that allows us to derive strict results for the avalanche-size distribution with input. We introduce the $(N, p)$ BB space as follows.

Definition A1.-Given positive integers $N$ and $M$, with $M>N$, define $p=(1 / M)$. The set $(N, p)$ BB consists of $(0,1)$ matrices of dimension $M \times N$. A $(0,1)$ matrix $\omega$ belongs to the set $(N, p) \mathrm{BB}$ if and only if, for all $j \in\{1,2, \ldots N\}, \sum_{i=1}^{M} \omega_{i, j}=1$, where $\omega_{i, j}$ denotes the $(i, j)$ th entry of $\omega$.

The set $(N, p)$ BB equipped with course sigma algebra is called the $(N, p)$ BB space. The elements of the set $(N, p)$ $\mathrm{BB}$ are referred to as configurations and typically denoted by $\omega, \omega^{\prime}$. 
The $(N, p)$ BB space is constructed to model neuronal networks. We interpret configuration $\omega$ as a record of the energy levels of all $N$ neurons at some moment of time. Each neuron occupies one of $M$ energy levels; if $\omega_{i, j}=1$, then the neuron $j$ is at the $i$ th energy level. Notice that, for all $j \in\{1,2, \ldots N\}$, there is a unique $i$ such that $\omega_{i, j}=1$; thus, we ensure that at any instant a neuron has one unique energy level. For $j \in\{1,2, \ldots N\}, E_{j}(\omega):=\inf _{i}\left\{\omega_{i, j}=1\right\}$ $\left(=\sup _{i}\left\{\omega_{i, j}=1\right\}\right) . E_{j}(\omega)$ documents the energy level of the $j$ th neuron. There is a linear ordering of the $M$ possible energy levels, which means $E_{j}(\omega)=M$ indicates that neuron $j$ is at the highest energy level.

For all $i \in\{1,2, \ldots M\}$, the number of neurons at the energy level $i$ is given by $Y_{i}(w):=\sum_{j=1}^{N} \omega_{i, j}$. We define the random variable $A_{N, p}$ that represents the avalanche size in the LM model. It is easy to see [50] that

$$
A_{N, p}:=\inf \left\{i \mid i \geq 0, \sum_{j=M-i}^{M} Y_{j} \leq i\right\} .
$$

We say that a configuration $\omega$ has generated $A_{N, p}(\omega)$ firings. The sets of all neurons fired during the avalanche $\mathcal{F}$ can be constructed from a configuration $\omega$ as

$$
\mathcal{F}(\omega)=\left\{j \mid \omega_{i, j}=1 \text { for some } i \geq M-A_{N, p}(\omega)\right\} .
$$

The set of not-fired neurons $\mathcal{N F}(\omega)$ can be found as

$$
\mathcal{N F}(\omega)=\left\{j \mid \omega_{i, j}=0, \quad \forall i \geq M-A_{N, p}(\omega)\right\} .
$$

We first equip $(N, p)$ BB space with the uniform measure, denoted by $P$. Intuitively, with the uniform measure every neuron has an equal probability of occupying any of the energy levels; also, there is no correlation between the energy levels of different neurons. It is defined as follows: $U^{M}$ is a uniformly distributed random variable taking values in $\{1,2, \ldots, M\}$, are $U_{j}^{M}$, $j \in\{1,2, \ldots, N\}$, iid copies of $U^{M}$ defined on some probability space $\Omega^{*}$. The map $C_{U}: \Omega^{*} \rightarrow(N, p) \mathrm{BB}$, $\theta \mapsto \omega$ is defined by $\omega_{i, j}(\theta)=\mathbb{1}_{i}\left(U_{j}^{M}(\theta)\right)$, where $\mathbb{1}$ is the indicator function. $P$ is the push forward measure of $C_{U}$ on $(N, p)$ BB. It was shown in Ref. [50] that

$$
P\left(A_{N, p}=k\right)=\left(\begin{array}{c}
N \\
k
\end{array}\right) p^{k}[1-(k+1) p]^{N-k}(k+1)^{k-1} .
$$

The next result enumerates the number of configurations satisfying a given property; it is used to prove results in later sections. It requires information about the number of labeled trees on $k$ vertices where a particular vertex has $a$ neighbors [72] (see Supplemental Material [73] for more details).
Theorem A1.-Define the set of configurations $\partial(N, k, a, p)$ as

$$
\begin{array}{r}
\partial(N, k, a, p)= \\
\left\{\omega \mid \omega \in(N, p) \mathrm{BB}, A_{N, p}(\omega)=k, Y_{M}(\omega)=a\right\} .
\end{array}
$$

Then $|\partial(N, k, a, p)|=\left(\begin{array}{c}k-1 \\ a-1\end{array}\right) k^{k-a}$.

\section{APPENDIX B: MODERATE INPUTS DURING AVALANCHE}

Now we construct the model for an avalanche with an input. Call the real number $\phi$ satisfying $0<\phi \leq 1$ the input strength. Let $\omega \in(N, p) \mathrm{BB}$, and $A_{N, p}(\omega)=o$. We define $\tau_{\phi}:(N, p) \mathrm{BB} \rightarrow(N, p) \mathrm{BB}, \omega \mapsto \omega^{\prime}=\tau_{\phi}(\omega)$. To define the function $\tau_{\phi}$ we first construct the set of neurons $\mathcal{E F}(\omega, \phi)$ that will be additionally firing due to the input. If $N-o \geq o \phi$, then $\mathcal{E F}(\omega, \phi)$ is a random subset of size $\lceil o \times \phi\rceil$ of $\mathcal{N F}(\omega)$. If $N-o<\lceil o \times \phi\rceil$, then $\mathcal{E F}(\omega)=\mathcal{N} \mathcal{F}(\omega)$. Now we can define $\omega^{\prime}=\tau_{\phi}(\omega)$ as follows: If $j \in \mathcal{E F}(\omega, \phi)$, then $\omega_{M, j}^{\prime}=1$ and $\omega_{i, j}^{\prime}=0$, $\forall i<M$. If $j \notin \mathcal{E F}(\omega, \phi)$, then $\omega_{i, j}^{\prime}=\omega_{i, j}, \forall i$.

$P_{\phi}^{E \text {,med }}$ is the push-forward measure of $P$ by $\tau_{\phi}$. Using Theorem A1, we can show the following.

Theorem B1.-Let $A_{N, p}$ be the avalanche random variable on the $(N, p) \mathrm{BB}$ space, and $\phi$ and $\tau_{\phi}$ are as above. For any positive integers $k, o$ satisfying $k \geq o+\lceil o \times \phi\rceil$, and for $\hat{p}=(p \times N / N-o)$, we have

$$
\begin{aligned}
& P\left(A_{N, p}\left(\tau_{\phi}(\omega)\right)=k \mid A_{N, p}(\omega)=o\right) \\
& =(\lceil o \times \phi\rceil)\left(\begin{array}{c}
N-\lceil o \times \phi\rceil-o \\
k-\lceil o \times \phi\rceil-o
\end{array}\right) \hat{p}^{k-\lceil o \times \phi\rceil-o} \\
& \quad \times[1-(k-o+1) \hat{p}]^{N-k}(k-o)^{k-1-\lceil o \times \phi\rceil-o} .
\end{aligned}
$$

We consider the above distribution as $N, k \rightarrow \infty, N \gg k$. We take $\phi>0$ to be a constant; i.e., we consider $\phi N \rightarrow \infty$. Thus, derived results are not expected to apply for the noinput regime with the measure $P$ even if one directly sets $\phi=0$. The following theorem is the central result of our study, demonstrating the 1.25 scaling (the proof is presented in Supplemental Material [73]).

Theorem B2.-We assume that $(N, \phi, k)$ satisfies

(i) $\phi>\left[1 / \log (k)^{\cdot 5}\right]$ and

(ii) $N \geq k^{2}$.

Then there exist positive constants $D_{1}$ and $D_{2}$ depending on $\phi$, such that

$$
D_{2} \leq \lim _{k \rightarrow \infty} \frac{P_{\phi}^{E, \text { med }}\left(A_{N, p}\left(\tau_{\phi}(\omega)\right)=k\right)}{k^{-1.25}} \leq D_{1} .
$$

Remark.-The conditions enforced on $(N, \phi, k)$ in Theorem 2 are sufficient but not necessary. For example, 
the condition $N>k^{2}$ is used to ensure terms like $e^{(o \phi / N-o-1)}$ are equal to 1 in the limit $N \rightarrow \infty$. The milder condition $N>o^{2}$ suffices for this.

\section{APPENDIX C: SMALL AND LARGE INPUTS DURING AVALANCHE}

Consider $\lambda \leq N$ an integer parameter. Let $\omega \in(N, p) \mathrm{BB}$, and we define $\omega^{\prime} \in(N, p)$ BB such that, if $j \leq \lambda, \omega_{M, j}^{\prime}=1$ and $\omega_{M, j}^{\prime}=0, \forall i<M$; if $j>\lambda, \omega_{i, j}^{\prime}=\omega_{i, j}, \quad \forall i$. Define $X_{N, p, \lambda}(\omega)=A_{N, p}\left(\omega^{\prime}\right)-\lambda$. We can derive the following.

Theorem C1. $-P\left(X_{N, p, \lambda}=k\right)=\left(\begin{array}{c}N-\lambda \\ k\end{array}\right) p^{k}(\lambda+1)(k+\lambda+$ $1)^{k-1}[1-(k+\lambda+1) p]^{N-\lambda-k}$.

If $\lambda=\lambda_{0}$, where $\lambda_{0}$ is some constant independent of $N$, we have a small input case. Using Theorem $\mathrm{C} 1$ and Stirling's formula, we show that, as $N$ and $k$ grow to infinity with $k / N \rightarrow 0, P\left(X_{N, p, \lambda}=k\right)=\Theta\left(k^{-1.5}\right)$.

For the large input case, we put $\lambda=\hat{\lambda}_{N}=\lambda_{0} \times N$, $\lambda_{0}<1$. We also demand that $\alpha \times\left(1+\lambda_{0}\right)<1$, which implies that there is massive external input during firing, which forces a proportion of the system to fire spontaneously. Now observe that $X_{N, p, \hat{\lambda}_{N}}$ has the distribution of a quasibinomial 1 distribution [74]. As $N \rightarrow \infty$, a quasibinomial 1 distribution approaches the generalized Poisson distribution [75], which is a type of Lagrangian distribution $[76,77]$. It has further been established that Lagrangian random variables approach the standard normal distribution under certain conditions [78]. Hence, we see that $X_{N, p, \hat{\lambda}_{N}}$ converges in the weak sense to a normal distribution.

\section{APPENDIX D: ADDITIONAL FIGURES}

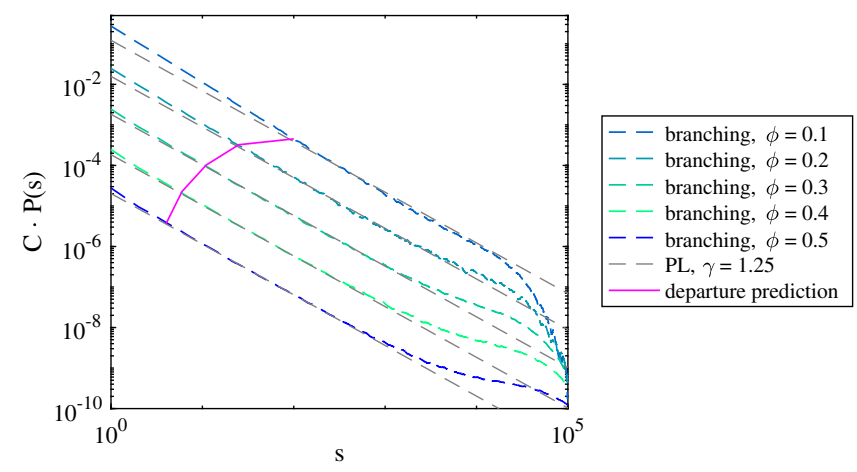

FIG. 6. Avalanche-size distributions in the branching model with various input strengths. Input strength $\phi$ and the model type are indicated in the legend. The magenta line indicates the analytic prediction for the onset of the power law with exponent -1.25 . System size $N=10^{5}$. To improve visibility, the distributions are shifted by multiplication with $c_{\phi}=10^{-10 \phi+1}$.

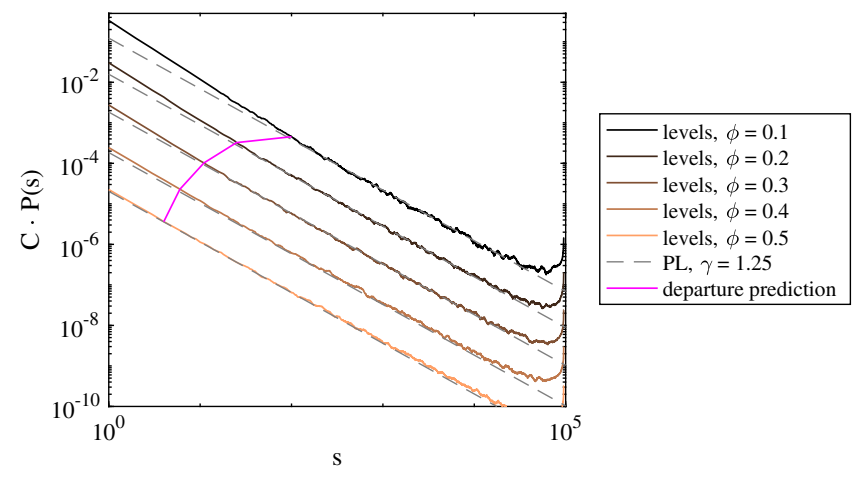

FIG. 7. Avalanche-size distributions in the levels model with various input strengths. Input strength $\phi$ and the model type are indicated in the legend. The magenta line indicates the analytic prediction for the onset of the power law with exponent -1.25 . System size $N=10^{5}$. To improve visibility, the distributions are shifted by multiplication with $c_{\phi}=10^{-10 \phi+1}$.

\section{APPENDIX E: STATISTICAL ANALYSIS OF POWER LAWS}

Here, we introduce rigorous statistical hypothesis testing and model selection for the scaling of avalanche-size distributions. The task of testing for power laws in the observed data is generally rather delicate, which is especially true in the present setting, where the theory suggests the avalanche-size distribution shows multifractal behavior. We benefit from the mathematical analysis of the LM that provides estimates for the intervals of each exponent prevalence. The power law with an exponent of approximately 1.5 is observed at small values of the avalanche size $s$. At $s \approx \phi^{-2}$, there is an onset of the 1.25 exponent power law that lasts at least until $s=\sqrt{N}$, where $N$ is the system size and $\phi$ is the input intensity. The upper bound of $s=\sqrt{N}$ is not a mathematically tight bound. To clarify Fig. 3: We are not claiming that there is a power law for $s$ close to the cutoff (we add more explanation of it to the text preceding Fig. 3). The reason for it is twofold: First, the analytic treatment omitted finite-size effects, and, second, in the BM there is a possibility for multiple inputs also during the "aftershock" that leads to a disproportional number of large avalanches.

For the BM simulations carried out with $\phi=0.2$ and $N=10^{5}$, we examine avalanches between 170 and 1700 . From different realizations of the network, we generate ten datasets of $5 \times 10^{5}$ avalanches each. We follow the approach suggested in Ref. [61] and perform both a goodness of fit testing and a comparison against alternative models.

For all ten datasets, and for the dataset formed by combining the whole data, we find the best-fit powerlaw model using the maximum likelihood approach $[61,62]$; the best-fit exponents are close to the infinite system theoretical limit of 1.25 from Eq. (1)—see the 
TABLE I. Goodness of power-law fit and model comparison with the log-normal distribution. The first column represents the dataset analyzed; the second column gives the best matched power-law exponent $\gamma$ obtained by maximum-likelihood methods; the third column gives $p$ values for a rejection of the powerlaw hypothesis, based on Kolmogorov-Smirnov distances [61]. The fourth column is a likelihood ratio of the power law over the log-normal (if larger than 1, the power law is preferred). The fifth column is the Akaike relative likelihood [79]. The indicated values demonstrate how likely the best-fit log-normal distribution is compared to the best-fit power-law distribution. The sixth column demonstrates the difference of BIC values (larger values correspond to stronger support of the power law over the lognormal). Each dataset consists of $5 \times 10^{5}$ avalanches, generated with $\phi=0.2, N=10^{5}$, and "all" combines the data from all ten datasets.

\begin{tabular}{lccccc}
\hline \hline Data & $\gamma$ & $p$ & $\mathcal{L}$ ratio & $\mathrm{AIC}_{\mathrm{LN} / \mathrm{PL}}$ & $\mathrm{BIC}_{\mathrm{LN}}-\mathrm{BIC}_{\mathrm{PL}}$ \\
\hline 1 & 1.26 & 0.76 & 3.67 & 0.0989 & 13.27 \\
2 & 1.27 & 0.55 & 1.22 & 0.308 & 11.00 \\
3 & 1.27 & 0.94 & 4.06 & 0.0929 & 13.39 \\
4 & 1.26 & 0.15 & 1.22 & 0.293 & 11.10 \\
5 & 1.27 & 0.51 & 1.82 & 0.217 & 11.70 \\
6 & 1.27 & 0.66 & 1.49 & 0.234 & 11.54 \\
7 & 1.27 & 0.54 & 1.35 & 0.256 & 11.37 \\
8 & 1.26 & 0 & 1.11 & 0.358 & 10.70 \\
9 & 1.27 & 0.37 & 1.65 & 0.227 & 11.61 \\
10 & 1.26 & 0.31 & 1.22 & 0.306 & 11.02 \\
All & 1.27 & 0.12 & $1.74 \times 10^{18}$ & $2.62 \times 10^{-19}$ & 13.27 \\
\hline \hline
\end{tabular}

TABLE II. Comparison of the power-law model against other models. For the same data as in Table I, we use Akaike information criterion to compare the power-law model versus competing models: $f_{1}(x)=C e^{\left\{a_{1} \log (x)+a_{2}[\log (x)]^{2}+a_{3}[\log (x)]^{3}\right\}}$ and $f_{2}(x)=C e^{a x^{b}}$, where in each case $C$ is a normalizing constant. $\mathrm{AIC}_{f_{i} / \mathrm{PL}}$ denotes a corresponding Akaike relative likelihood of $f_{i}$ compared to a power law. In all cases, the power law is a preferred model. We also compare against the thresholded OrnsteinUhlenbeck process: $d x=-(x / 80) d t+0.625 \times d W_{t}$ at various thresholds; the relative likelihood for the best parameters and threshold is $\mathrm{AIC}_{\mathrm{OU} / \mathrm{PL}}$.

\begin{tabular}{lllc}
\hline \hline Data & $\mathrm{AIC}_{\mathrm{OU} / \mathrm{PL}}$ & $\mathrm{AIC}_{f_{1} / \mathrm{PL}}$ & $\mathrm{AIC}_{f_{2} / \mathrm{PL}}$ \\
\hline 1 & $8.36 \times 10^{-13}$ & 0.00414 & 0.000817 \\
2 & $1.89 \times 10^{-9}$ & 0.00054 & 0.000198 \\
3 & $3.75 \times 10^{-13}$ & 0.000837 & 0.000597 \\
4 & $1.30 \times 10^{-9}$ & 0.00157 & $2.10 \times 10^{-7}$ \\
5 & $1.60 \times 10^{-12}$ & 0.00241 & 0.000198 \\
6 & $2.94 \times 10^{-12}$ & 0.00056 & 0.000829 \\
7 & $8.84 \times 10^{-12}$ & 0.00122 & $2.00 \times 10^{-4}$ \\
8 & $7.96 \times 10^{-9}$ & 0.0383 & 0.000145 \\
9 & $1.08 \times 10^{-9}$ & 0.00634 & $7.96 \times 10^{-9}$ \\
10 & $6.19 \times 10^{-6}$ & 0.00189 & $3.22 \times 10^{-26}$ \\
\hline \hline
\end{tabular}

second column in Table I. We measure the KolmogorovSmirnov distance and perform the test for a rejection of the power-law hypothesis based on surrogate data [61]. We cannot reject the power-law hypothesis for all but the eighth dataset (see the third column in Table I). We compare the best-fit power law with the best-fit truncated log-normal [56], which is usually the strongest alternative model. The likelihood ratio test, Akaike criteria, and Bayesian information criteria show that the power law is a more plausible model than the truncated log-normal for all datasets; see Table I. We consider additional possibilities for model comparison: exponential distribution, which is shown to be a suitable alternative model [57], and other models investigated for a model comparison against power laws [58,59]. We compare the models using the Akaike relative likelihood parameter [79]. As a different type of alternative model, we also consider excursion statistics above thresholds for certain Ornstein-Uhlenbeck processes. It is observed [60] that such excursions not only resemble neuronal signals, but also produce power-law-like distributions with the exponent related to the chosen threshold. We present comparison results for the most significant or most widely used models in Table II. In all the cases studied, the power law proved to be a much more probable choice.

[1] B. Gutenberg and C. F. Richter, Magnitude and Energy of Earthquakes, Annali di Geofisica 9, 1 (1956).

[2] D. Sornette, Critical Phenomena in Natural Sciences: Chaos, Fractals, Self-Organization and Disorder: Concepts and Tools (Springer, New York, 2006).

[3] J. Beggs and D. Plenz, Neuronal Avalanches in Neocortical Circuits, J. Neurosci. 23, 11167 (2003).

[4] A. Levina and V. Priesemann, Subsampling Scaling, Nat. Commun. 8, 15140 (2017).

[5] V. Priesemann, M. Valderrama, M. Wibral, and M. Le Van Quyen, Neuronal Avalanches Differ from Wakefulness to Deep Sleep_Evidence from Intracranial Depth Recordings in Humans, PLoS Comput. Biol. 9, e1002985 (2013).

[6] E. Tagliazucchi, P. Balenzuela, D. Fraiman, and D. R. Chialvo, Criticality in Large-Scale Brain FMRI Dynamics Unveiled by a Novel Point Process Analysis, Front. Physiol. 3, 15 (2012).

[7] O. Shriki, J. Alstott, F. Carver, T. Holroyd, R. N. Henson, M. L. Smith, R. Coppola, E. Bullmore, and D. Plenz, Neuronal Avalanches in the Resting Meg of the Human Brain, J. Neurosci. 33, 7079 (2013).

[8] T. Petermann, T. C. Thiagarajan, M. A. Lebedev, M. A. Nicolelis, D. R. Chialvo, and D. Plenz, Spontaneous Cortical Activity in Awake Monkeys Composed of Neuronal Avalanches, Proc. Natl. Acad. Sci. U.S.A. 106, 15921 (2009).

[9] N. Friedman, S. Ito, B. A. W. Brinkman, M. Shimono, R. E. L. DeVille, K. A. Dahmen, J. M. Beggs, and T. C. Butler, Universal Critical Dynamics in High Resolution 
Neuronal Avalanche Data, Phys. Rev. Lett. 108, 208102 (2012).

[10] A. Klaus, S. Yu, and D. Plenz, Statistical Analyses Support Power Law Distributions Found in Neuronal Avalanches, PLoS One 6, e19779 (2011).

[11] C. G. Langton, Computation at the Edge of Chaos: Phase Transitions and Emergent Computation, Physica (Amsterdam) 42D, 12 (1990).

[12] N. Bertschinger and T. Natschläger, Real-Time Computation at the Edge of Chaos in Recurrent Neural Networks, Neural Comput. 16, 1413 (2004).

[13] J. Boedecker, O. Obst, J. T. Lizier, N. M. Mayer, and M. Asada, Information Processing in Echo State Networks at the Edge of Chaos, Theory Biosci. 131, 205 (2012).

[14] W. L. Shew, H. Yang, T. Petermann, R. Roy, and D. Plenz, Neuronal Avalanches Imply Maximum Dynamic Range in Cortical Networks at Criticality, J. Neurosci. 29, 15595 (2009).

[15] O. Kinouchi and M. Copelli, Optimal Dynamical Range of Excitable Networks at Criticality, Nat. Phys. 2, 348 (2006).

[16] V. Priesemann and O. Shriki, Can a Time Varying External Drive Give Rise to Apparent Criticality in Neural Systems?, PLoS Comput. Biol. 14, e1006081 (2018).

[17] J. M. Beggs and N. Timme, Being Critical of Criticality in the Brain, Front. Oral Physiol. 3, 163 (2012).

[18] C. Bedard, H. Kroger, and A. Destexhe, Does the 1/f Frequency-Scaling of Brain Signals Reflect Self-Organized Critical States?, Phys. Rev. Lett. 97, 118102 (2006).

[19] P. Bak, C. Tang, and K. Wiesenfeld, Self-Organized Criticality: An Explanation of $1 / f$ Noise, Phys. Rev. Lett. 59, 381 (1987).

[20] J. A. Bonachela and M. A. Muñoz, Self-Organization without Conservation: True or Just Apparent ScaleInvariance?, J. Stat. Mech.: Theory Exp. (2009) P09009.

[21] G. Pruessner, Self-Organised Criticality: Theory, Models and Characterisation (Cambridge University Press, Cambridge, England, 2012).

[22] R. Dickman, M. A. Muñoz, A. Vespignani, and S. Zapperi, Paths to Self-Organized Criticality, Braz. J. Phys. 30, 27 (2000).

[23] R. Dickman, A. Vespignani, and S. Zapperi, Self-Organized Criticality as an Absorbing-State Phase Transition, Phys. Rev. E 57, 5095 (1998).

[24] N. W. Watkins, G. Pruessner, S. C. Chapman, N. B. Crosby, and H. J. Jensen, 25 Years of Self-Organized Criticality: Concepts and Controversies, Space Sci. Rev. 198, 3 (2016).

[25] P. Bak, How Nature Works: The Science of Self-Organized Criticality (Springer Verlag, Berlin, 1999).

[26] Á. Corral, C. J. Pérez, A. Díaz-Guilera, and A. Arenas, SelfOrganized Criticality and Synchronization in a Lattice Model of Integrate-and-Fire Oscillators, Phys. Rev. Lett. 74, 118 (1995).

[27] S. Zapperi, K. B. Lauritsen, and H. E. Stanley, SelfOrganized Branching Processes: Mean-Field Theory for Avalanches, Phys. Rev. Lett. 75, 4071 (1995).

[28] A. V. M. Herz and J. J. Hopfield, Earthquake Cycles and Neural Reverberations: Collective Oscillations in Systems with Pulse-Coupled Threshold Elements, Phys. Rev. Lett. 75, 1222 (1995).
[29] C. W. Eurich, J. M. Herrmann, and U. Ernst, Finite-Size Effects of Avalanche Dynamics, Phys. Rev. E 66, 066137 (2002).

[30] L. de Arcangelis, C. Perrone-Capano, and H. J. Herrmann, Self-Organized Criticality Model for Brain Plasticity, Phys. Rev. Lett. 96, 028107 (2006).

[31] A. Levina, J. M. Herrmann, and T. Geisel, Dynamical Synapses Causing Self-Organized Criticality in Neural Networks, Nat. Phys. 3, 857 (2007).

[32] A. Levina, J. M. Herrmann, and T. Geisel, Phase Transitions towards Criticality in a Neural System with Adaptive Interactions, Phys. Rev. Lett. 102, 118110 (2009).

[33] S. Scarpetta and A. de Candia, Alternation of Up and Down States at a Dynamical Phase-Transition of a Neural Network with Spatiotemporal Attractors, Front. Syst. Neurosci. 8, 88 (2014).

[34] S. di Santo, P. Villegas, R. Burioni, and M. A. Muñoz, Landau-Ginzburg Theory of Cortex Dynamics: Scale-Free Avalanches Emerge at the Edge of Synchronization, Proc. Natl. Acad. Sci. U.S.A. 115, E1356 (2018).

[35] M. A. Munoz, Colloquium: Criticality and Dynamical Scaling in Living Systems, Rev. Mod. Phys. 90, 031001 (2018).

[36] D. Millman, S. Mihalas, A. Kirkwood, and E. Niebur, Self-Organized Criticality Occurs in Non-Conservative Neuronal Networks during "Up" States, Nat. Phys. 6, 801 (2010).

[37] M. Martinello, J. Hidalgo, A. Maritan, S. Di Santo, D. Plenz, and M. A. Muńoz, Neutral Theory and Scale-Free Neural Dynamics, Phys. Rev. X 7, 041071 (2017).

[38] V. Priesemann, M. Wibral, M. Valderrama, R. Pröpper, M. Le Van Quyen, T. Geisel, J. Triesch, D. Nikolić, and M. H. J. Munk, Spike Avalanches in vivo Suggest a Driven, Slightly Subcritical Brain State, Front. Syst. Neurosci. 8, 108 (2014).

[39] R. N. Gutenkunst, J. J. Waterfall, F. P. Casey, K. S. Brown, C. R. Myers, and J. P. Sethna, Universally Sloppy Parameter Sensitivities in Systems Biology Models, PLoS Comput. Biol. 3, e189 (2007).

[40] M. Wilkinson, R. Guichardaz, M. Pradas, and A. Pumir, Power-Law Distributions in Noisy Dynamical Systems, Europhys. Lett. 111, 50005 (2015).

[41] E.S. Palamarchuk, An Analytic Study of the OrnsteinUhlenbeck Process with Time-Varying Coefficients in the Modeling of Anomalous Diffusions, Automation and Remote Control (English Translation) 79, 289 (2018).

[42] B. J. West and N. Scafetta, Nonlinear Dynamical Model of Human Gait, Phys. Rev. E 67, 051917 (2003).

[43] J. Wilting and V. Priesemann, Inferring Collective Dynamical States from Widely Unobserved Systems, Nat. Commun. 9, 2325 (2018).

[44] S. Yu, T. L. Ribeiro, C. Meisel, S. Chou, A. Mitz, R. Saunders, and D. Plenz, Maintained Avalanche Dynamics during Task-Induced Changes of Neuronal Activity in Nonhuman Primates, eLife 6, e27119 (2017).

[45] C. Haldeman and J. M. Beggs, Critical Branching Captures Activity in Living Neural Networks and Maximizes the Number of Metastable States, Phys. Rev. Lett. 94, 058101 (2005). 
[46] A. Levina, J. M. Herrmann, and M. Denker, Critical Branching Processes in Neural Networks, Proc. Appl. Math. Mech. 7, 1030701 (2007).

[47] A. Levina, A Mathematical Approach to Self-Organized Criticality in Neural Networks, Ph. D. thesis, Nieders. Staatsu. Universitätsbibliothek Göttingen, 2008.

[48] A. Levina and J. M. Herrmann, The Abelian Distribution, Stochastics Dynamics 14, 1450001 (2014).

[49] M. Denker and A. Levina, Avalanche dynamics, Stochastics Dynamics 16, 1660005 (2016).

[50] M. Denker and A. Rodrigues, Ergodicity of Avalanche Transformations, Dyn. Stab. Syst. 29, 517 (2014).

[51] D. Dhar, The Abelian Sandpile and Related Models, Physica (Amsterdam) 263A, 4 (1999).

[52] A. Levina, U. Ernst, and J. M. Herrmann, Criticality of Avalanche Dynamics in Adaptive Recurrent Networks, Neurocomput. 70, 1877 (2007).

[53] R. V. Williams-García, M. Moore, J. M. Beggs, and G. Ortiz, Quasicritical Brain Dynamics on a Nonequilibrium Widom Line, Phys. Rev. E 90, 062714 (2014).

[54] $f(n)=\Theta(g(n))$ if $\exists k_{1}>0, \exists k_{2}>0, \exists n_{0}$ such that $\forall n>n_{0}$ we have $k_{1} \cdot g(n) \leq f(n) \leq k_{2} \cdot g(n)$.

[55] D. Harte, Multifractals: Theory and Applications (CRC, Boca Raton, FL, 2001).

[56] Á. Corral and Á. González, Power-Law Distributions in Geoscience Revisited, arXiv:1810.07868 .

[57] J. Touboul and A. Destexhe, Can Power-Law Scaling and Neuronal Avalanches Arise from Stochastic Dynamics?, PLoS One 5, e8982 (2010).

[58] R. Ton and A. Daffertshofer, Model Selection for Identifying Power-Law Scaling, NeuroImage 136, 215 (2016).

[59] A. Daffertshofer, R. Ton, M. L. Kringelbach, M. Woolrich, and G. Deco, Distinct Criticality of Phase and Amplitude Dynamics in the Resting Brain, NeuroImage 180, 442 (2018).

[60] P. Villegas, S. di Santo, R. Burioni, and M. A. Muñoz, Timeseries Thresholding and the Definition of Avalanche Size, arXiv:1902.10465.

[61] A. Clauset, C. R. Shalizi, and M. E. Newman, Power-Law Distributions in Empirical Data, SIAM Rev. 51, 661 (2009).

[62] H. Bauke, Parameter Estimation for Power-Law Distributions by Maximum Likelihood Methods, Eur. Phys. J. B 58, 167 (2007).

[63] K. Christensen and N. R. Moloney, Complexity and Criticality (World Scientific, Singapore, 2005), Vol. 1.

[64] D. Bonamy, S. Santucci, and L. Ponson, Crackling Dynamics in Material Failure as the Signature of a SelfOrganized Dynamic Phase Transition, Phys. Rev. Lett. 101, 045501 (2008).
[65] L. Laurson, S. Santucci, and S. Zapperi, Avalanches and Clusters in Planar Crack Front Propagation, Phys. Rev. E 81, 046116 (2010).

[66] G. Caldarelli, F. D. Di Tolla, and A. Petri, Self-Organization and Annealed Disorder in a Fracturing Process, Phys. Rev. Lett. 77, 2503 (1996).

[67] M. Talamali, V. Petäjä, D. Vandembroucq, and S. Roux, Avalanches, Precursors, and Finite-Size Fluctuations in a Mesoscopic Model of Amorphous Plasticity, Phys. Rev. E 84, 016115 (2011).

[68] S. Mossa, M. Barthelemy, H. E. Stanley, and L. A. N. Amaral, Truncation of Power Law Behavior in Scale-Free Network Models due to Information Filtering, Phys. Rev. Lett. 88, 138701 (2002).

[69] R. V. Williams-García, J. M. Beggs, and G. Ortiz, Unveiling Causal Activity of Complex Networks, Europhys. Lett. 119, 18003 (2017).

[70] W. L. Shew, W. P. Clawson, J. Pobst, Y. Karimipanah, N. C. Wright, and R. Wessel, Adaptation to Sensory Input Tunes Visual Cortex to Criticality, Nat. Phys. 11, 659 (2015); D. B. Larremore, W. L. Shew, E. Ott, F. Sorrentino, and J. G. Restrepo, Inhibition Causes Ceaseless Dynamics in Networks of Excitable Nodes, Phys. Rev. Lett. 112, 138103 (2014).

[71] M. Yaghoubi, T. de Graaf, J. G. Orlandi, F. Girotto, M. A. Colicos, and J. Davidsen, Neuronal Avalanche Dynamics Indicates Different Universality Classes in Neuronal Cultures, Sci. Rep. 8, 3417 (2018).

[72] J. W. Moon, Various Proofs of Cayley's Formula for Counting Trees, in A Seminar on Graph Theory (Holt, Rinehart and Winston, New York, 1967), p. 78.

[73] See Supplemental Material at http://link.aps.org/ supplemental/10.1103/PhysRevX.9.021062 for formal proofs of the presented theorems.

[74] P. C. Consul, A Simple Urn Model Dependent upon Predetermined Strategy, Sankhyā: The Indian Journal of Statistics, Series B 36, 391 (1974).

[75] P. C. Consul and G. C. Jain, A Generalization of the Poisson Distribution, Technometrics 15, 791 (1973).

[76] P. Consul and L. Shenton, Use of Lagrange Expansion for Generating Discrete Generalized Probability Distributions, SIAM J. Appl. Math. 23, 239 (1972).

[77] P. C. Consul and F. Famoye, Lagrangian Probability Distributions (Springer, New York, 2006).

[78] P. C. Consul and L. Shenton, Some Interesting Properties of Lagrangian Distributions, Commun. Stat., Theory Methods 2, 263 (1973).

[79] K. P. Burnham and D. R. Anderson, Model Selection and Multimodel Inference. A Practical Information-Theoretic Approach (Springer, New York, 2002). 